\title{
ALTERAÇÕES EM ATRIBUTOS QUÍMICOS DE UM SOLO SUBMETIDO À ADUBAÇÃO E CULTIVADO COM VIDEIRA 'NIAGARA ROSADA'
}

\author{
LUIZ ANTONIO JUNQUEIRA TEIXEIRA², MARCO ANTONIO TECCHIO², \\ MARA FERNANDES MOURA ${ }^{2}$, MAURILO MONTEIRO TERRA ${ }^{2}$, \\ ERASMO JOSÉ PAIOLI PIRES ${ }^{2}$, JOSÉ LUIZ HERNANDES ${ }^{2}$
}

RESUMO - Com o objetivo de avaliar alterações em atributos químicos do solo, causadas pela adubação com NPK em videira 'Niagara Rosada', coletaram-se amostras de solo provenientes de um experimento de campo executado em Louveira (SP), durante quatro ciclos de produção. O delineamento experimental foi em blocos ao acaso, no esquema fatorial fracionado $1 / 2\left(4^{3}\right)$, com tratamentos constituídos pelas combinações de doses de $\mathrm{N}(0 ; 65 ; 130$ e $195 \mathrm{~kg} / \mathrm{ha}$ de $\mathrm{N}), \mathrm{P}\left(0 ; 60 ; 120\right.$ e $180 \mathrm{~kg} / \mathrm{ha}$ de $\left.\mathrm{P}_{2} \mathrm{O}_{5}\right)$ e $\mathrm{K}(0 ; 45 ; 90$ e $135 \mathrm{~kg} / \mathrm{ha}$ de $\mathrm{K}_{2} \mathrm{O}$ ) aplicados em cada ciclo de produção. As doses de N, P e K foram aplicadas em forma fracionada, empregando-se como fontes $\mathrm{NH}_{4} \mathrm{NO}_{3}$, superfosfato triplo e $\mathrm{KCl}$, respectivamente. $\mathrm{Na}$ implantação do experimento, em agosto de 2005, e após quatro safras, em novembro de 2008, foram coletadas amostras de solo de 0 a $20 \mathrm{~cm}$ e de 20 a $40 \mathrm{~cm}$ de profundidade, determinando-se valores de $\mathrm{pH}\left(\mathrm{CaCl}_{2}\right)$, o teor de matéria orgânica, $\mathrm{P}, \mathrm{K}, \mathrm{Ca}, \mathrm{Mg}, \mathrm{H}+\mathrm{Al}$ e calculada a soma de bases, CTC e saturação por bases. Avaliaramse, também, a produtividade e as características físicas dos cachos e das bagas. A adubação nitrogenada em videira determinou incrementos significativos na acidez do solo. Doses baixas de $\mathrm{Pe} \mathrm{K}$ foram suficientes para manter o teor de $\mathrm{P}$ e de $\mathrm{K}$ do solo no mesmo nível da amostragem inicial, após quatro ciclos de produção. A produtividade e as características físicas dos cachos não foram influenciadas pela adubação com $\mathrm{N}, \mathrm{P}$ e K. A adubação com N, P e K em vinhedos com alta disponibilidade de nutrientes no solo, condição comum na região de Louveira e Jundiaí, implica prejuízo econômico ao produtor, eleva os teores de nutrientes no solo acima das necessidades da cultura, podendo, ainda, aumentar o risco de contaminação ambiental.

Termos para indexação: análise de solo, uva, nitrogênio, fósforo, potássio.

\section{CHANGES ON SOME SOIL CHEMICAL PROPERTIES DUE TO NPK FERTILIZATION FOR 'NIGARA ROSADA' GRAPEVINE}

\begin{abstract}
Soil samples were analyzed with the objective of investigating changes on some soil chemical properties due to NPK fertilization of 'Niagara Rosada' grapevine. The samples were collected in a field experiment carried out in Louveira, Brazil, during four crop seasons. The experimental design was an incomplete NPK factorial $1 / 2\left(4^{3}\right)$. The treatments were rates of $\mathrm{N}\left(0,65,130\right.$, and $195 \mathrm{~kg} / \mathrm{ha}$ of N as $\left.\mathrm{NH}_{4} \mathrm{NO}_{3}\right)$, $\mathrm{P}\left(0,60,120\right.$, and $180 \mathrm{~kg} / \mathrm{ha}$ of $\mathrm{P}_{2} \mathrm{O}_{5}$ as triple superphosphate) and $\mathrm{K}\left(0,45,90\right.$, and $135 \mathrm{~kg} / \mathrm{ha}$ of $\mathrm{K}_{2} \mathrm{O}$ as $\mathrm{KCl}$ ) applied at each crop season. Nutrient $(\mathrm{P}, \mathrm{K}, \mathrm{Ca}$ and $\mathrm{Mg}$ ) availability some chemical properties (organic matter, $\mathrm{pH} \mathrm{CaCl}, \mathrm{H}+\mathrm{Al}$ and base saturation) were evaluated through samples carried out in the experiment implantation (August, 2005) and after four production cycles (November, 2008). Soil samples were collected from layers of $0-20 \mathrm{~cm}$ and $20-40 \mathrm{~cm}$. Fruit yield and some physical characteristics of bunches and berries were evaluated. Soil $\mathrm{pH}$ decreased with increasing in $\mathrm{N}$ rates. Low rates of $\mathrm{P}$ and $\mathrm{K}$ were sufficient to maintain soil $\mathrm{P}$ and $\mathrm{K}$ at the same concentration as found prior to the experiment. Grapevine production was not affected by NPK fertilization. The application of NPK fertilizers in vineyards with soils with high nutrient availability implies economic losses and environmental contamination risk.
\end{abstract}

Index terms: soil analysis, grape, nitrogen, phosphorus, potassium.

\footnotetext{
${ }^{1}$ (Trabalho 139-10). Recebido em: 01-06-2010. Aceito para publicação em: 12-07-2011. Trabalho realizado com apoio da FAPESP (Processo: 05/03410-9).

${ }^{2}$ Pesquisador do Centro de Solos e Recursos Ambientais do Instituto Agronômico (IAC). Cx. Postal 28, 13012-970, Campinas-SP. teixeira@iac.sp.gov.br

${ }^{3}$ Pesquisadores do Centro de Fruticultura do Instituto Agronômico (IAC). E-mails: tecchio@iac.sp.gov.br ; mouram@iac.sp.gov.br; mmterra@iac.sp.gov.br; ejppires@iac.sp.gov.br; jlhernandes@iac.sp.gov.br
} 


\section{INTRODUÇÃO}

O maior produtor nacional de uva para mesa é o Estado de São Paulo, com produção de 189,7 mil toneladas. A cultivar Niagara Rosada responde por $89,1 \%$ das videiras e $48,2 \%$ da produção de uva no Estado. Na região leste de São Paulo, a Regional Agrícola de Campinas participa com cerca de $77 \%$ da produção de 'Niagara Rosada' do Estado, destacando-se os municípios de Jundiaí, Louveira, Vinhedo e Itupeva (INSTITUTO DE ECONOMIA AGRÍCOLA, 2010).

Entre as práticas culturais visando ao aumento em produtividade e à qualidade da uva, merece destaque a aplicação balanceada de nutrientes mediante adubações equilibradas, baseadas em análises químicas de solo e de folha. De acordo com Fráguas et al. (2002) e Terra et al. (2003), os vinhedos brasileiros poderiam alcançar elevadas produções com frutos de melhor qualidade caso fossem adubados adequadamente. Fregoni (1980) mencionou que a nutrição adequada das plantas promove, além do aumento em produtividade, melhoras na maturação, formato, firmeza da polpa, cor, tamanho e uniformidade dos bagos, havendo, também, efeito na concentração de açúcares e acidez dos frutos.

A fertilidade do solo influi no crescimento e na distribuição do sistema radicular, no desenvolvimento da parte aérea e a na composição dos frutos (LANYON et al., 2004). Entretanto, estes autores afirmaram que há poucas informações objetivas que relacionem atributos químicos do solo com o desempenho das plantas. No caso de excesso de N, a videira torna-se muito vigorosa, prolongando o período de crescimento vegetativo e retardando o amadurecimento dos frutos (HILBERT et al., 2003). Este quadro leva à formação de bagas aquosas e moles, à redução da fertilidade de gemas e ao maior sombreamento, formando cachos menores e com maior acidez (FREGONI, 1980). O excesso de P aumenta a acidez do mosto, reduz o teor de suco da polpa e pode induzir à deficiência de Fe e Zn (FREGONI, 1980). Quando a relação $\mathrm{K}_{2} \mathrm{O} / \mathrm{MgO}$ for superior a 10 , o excesso de K pode provocar dessecamento da ráquis, pela menor absorção de cálcio e magnésio, antecipação na entrada do repouso vegetativo e atraso na retomada da atividade vegetativa no ciclo seguinte, bem como sintomas de deficiência de $\mathrm{Mg}$ (TAGLIAVINI et al., 1996).

$\mathrm{Na}$ literatura, são encontrados inúmeros trabalhos com adubação de videira nos quais se avaliaram respostas das plantas relacionadas ao vigor, à produtividade, à qualidade dos cachos e aos teores foliares de nutrientes (CONRADIE; SAAYMAN,
1989A; CONRADIE; SAAYMAN, 1989B; TERRA 1990; DAL BÓ, 1992; REUVENI et al. 1994; LINSENMEIER et al., 2008; NEILSEN et al., 2010). No entanto, são escassas as publicações nas quais seja avaliado o efeito da adubação nas características químicas do solo. Estas informações são importantes, tendo em vista que, na região de Jundiaí, foram observados problemas relacionados à fertilidade do solo, seja pelo excesso, seja por deficiência de nutrientes, decorrentes do cultivo da videira 'Niagara Rosada' (TECCHIO et al., 2006).

A partir de um levantamento de aspectos agronômicos e do estado nutricional de vinhedos da região de Jundiaí, Tecchio et al. (2006) verificaram que, embora a prática da adubação seja realizada pela maioria dos viticultores na região de Jundiaí, eles a fazem de forma inadequada, baseando-se em orientações empíricas, sem levar em consideração resultados de análise de solo ou as necessidades nutricionais das plantas. Os autores observaram desequilíbrio entre os teores de cálcio, magnésio e potássio no solo, afetando de maneira significativa a produtividade da videira 'Niagara Rosada' em vinhedos adubados de forma inadequada. Tonietto (1994), em Bento Gonçalves, Rio Grande do Sul, e Sharma et al. (2003), na Índia, também constataram desequilíbrios nutricionais decorrentes do excesso de adubação nos vinhedos.

$\mathrm{O}$ risco de danos ambientais induzido pelo uso inadequado de fertilizantes é uma preocupação que atualmente vem ganhando importância. Segundo Grant (2010), a análise de solo, visando a predizer a disponibilidade de nutrientes, é o primeiro passo para a determinação da dose adequada de fertilizantes que minimize perdas e contaminação ambiental. Outrossim, as práticas de manejo que conciliam a adubação com a demanda da cultura, em termos de dose e época de aplicação, podem aumentar a eficiência dos fertilizantes e reduzir os impactos ambientais.

O objetivo deste trabalho foi estudar alterações em atributos químicos de um solo em condições típicas de cultivo de videira 'Niagara Rosada' na região de Jundiaí, bem como a produção, em função da adubação NPK durante quatro ciclos de produção

\section{MATERIAL E MÉTODOS}

O experimento foi instalado em Louveira-SP (2304'44"S; 46 55'09'O), numa altitude em torno de $710 \mathrm{~m}$, apresentando médias anuais de $1.400 \mathrm{~mm}$ de precipitação pluvial, temperatura média de $19,5^{\circ} \mathrm{C}$ e umidade relativa do ar de $71 \%$. O clima, segundo a classificação de Köeppen, é Cwa (mesotérmico de 
inverno seco). De acordo com o Mapa Pedológico do Estado de São Paulo (OLIVEIRA et al., 1999), as áreas experimentais estão incluídas na unidade de mapeamento dos ARGISSOLOS VERMELHOAMARELOS (PVA) Distróficos, A moderado, textura argilosa e média cascalhenta/argilosa cascalhenta, fase rochosa, relevo forte ondulado e montanhoso. $\mathrm{Na}$ Tabela 1, são apresentados alguns atributos químicos do solo da área experimental, obtidos em amostragem prévia à instalação do experimento. Essas condições edafoclimáticas são bastante comuns nos vinhedos da região de Louveira e Jundiaí.

$\mathrm{O}$ experimento foi realizado em meio a uma área de produção comercial, num vinhedo de 'Niagara Rosada' enxertada sobre IAC 766 'Campinas', sustentada em espaldeira, com espaçamento de 1,7 X $0,9 \mathrm{~m}$. Os porta-enxertos foram plantados em julho de 2004, efetuando-se a enxertia em agosto de 2005. Previamente à instalação do experimento, a área já vinha sendo cultivada com videira. Esta área foi escolhida para instalação do experimento por ser bastante representativa das condições de cultivo regionais.

Empregou-se o delineamento experimental de blocos ao acaso, no esquema fatorial fracionado $1 / 2$ $\left(4^{3}\right)$, com os tratamentos formados pelas combinações de doses de $\mathrm{N}(0 ; 65 ; 130$ e $195 \mathrm{~kg} / \mathrm{h}$ a de $\mathrm{N}), \mathrm{P}(0 ; 60$; 120 e $180 \mathrm{~kg} / \mathrm{ha}$ de $\left.\mathrm{P}_{2} \mathrm{O}_{5}\right)$ e K $(0 ; 45 ; 90$ e $135 \mathrm{~kg} / \mathrm{ha}$ de $\mathrm{K}_{2} \mathrm{O}$ ) aplicadas em cada ciclo de produção. Estas doses foram estabelecidas buscando-se estabelecer uma curva de resposta à aplicação de N, P e K, sendo a dose máxima aproximadamente $50 \%$ superior à recomendada por Terra et al. (1997) e incluindo a variação de adubação observada nos vinhedos da região. As unidades experimentais foram compostas por quinze plantas (três linhas com cinco plantas), sendo três da linha central consideradas para as avaliações (plantas úteis).

Realizou-se a aplicação dos fertilizantes em sulcos ao lado das plantas, fracionando as doses de $\mathrm{N}$, P e K de acordo com o seguinte cronograma: $100 \%$ da dose de $\mathrm{P}_{2} \mathrm{O}_{5}$ e $50 \%$ do $\mathrm{K}_{2} \mathrm{O}$ aplicados antes da poda; $50 \%$ da dose de $\mathrm{N}$ aplicado vinte dias após a poda e $50 \%$ da dose de $\mathrm{K}_{2} \mathrm{O}$ e $50 \%$ da dose de $\mathrm{N}$ aplicado na fase de chumbinho à $1 / 2$ baga. $O$ parcelamento das doses dos fertilizantes seguiu a recomendação de Terra et al. (1997). As fontes de N, P e K utilizadas foram, respectivamente, nitrato de amônio, superfosfato triplo e cloreto de potássio. Adicionalmente, aplicaram-se $2 \mathrm{~kg} /$ planta de cama de frango antes da poda, em cada ciclo de produção, seguindo a dose comumente utilizada pelos viticultores da região. $\mathrm{O}$ uso da cama de aviário nas condições do experimento adicionou, em média, $160 \mathrm{~kg} / \mathrm{ha}$ de N, $170 \mathrm{~kg} / \mathrm{ha}$ de $\mathrm{P}_{2} \mathrm{O}_{5}$ e $140 \mathrm{~kg} / \mathrm{ha}$ de $\mathrm{K}_{2} \mathrm{O}$ por aplicação. Parte destes nutrientes tornou-se disponível pela mineralização da cama de aviário.

A primeira poda, realizada na altura do segundo arame da espaldeira, ocorreu no mesmo ano da enxertia, em dezembro de 2005. Depois da poda, utilizou-se calciocianamida (20\%) para uniformizar a brotação, sendo a primeira colheita realizada em abril de 2006. A segunda poda (curta com cordão esporonado) foi feita em agosto de 2006. Em dezembro, fez-se a segunda colheita. Em janeiro de 2007, efetuou-se novamente poda temporã próxima ao segundo arame da espaldeira, sendo a terceira colheita realizada em junho. Para a quarta colheita da área experimental, realizada em dezembro de 2007, fez-se a poda de inverno em setembro. Para cada ciclo de produção, realizou-se o parcelamento das adubações conforme descrito anteriormente. Os tratos culturais seguiram as práticas recomendadas para a região de Jundiaí (BRAGA, 1988).

Por meio da amostragem de solo inicial realizada em agosto de 2005 e da coleta feita em março de 2008, caracterizaram-se as unidades experimentais de modo individualizado, permitindo que se estimasse a evolução da fertilidade em função dos tratamentos de adubação. Foram coletadas amostras de solo compostas (seis subamostras) nas camadas de 0 a $20 \mathrm{~cm}$ e de 20 a $40 \mathrm{~cm}$, na área de aplicação dos fertilizantes, aproximadamente 20 a $30 \mathrm{~cm}$ do caule das videiras. Realizaram-se análises químicas de $\mathrm{pH}$ em $\mathrm{CaCl}_{2}$, teor de matéria orgânica, $\mathrm{P}$ pelo método da resina, $\mathrm{K}, \mathrm{Ca}$ e $\mathrm{Mg}$ trocável, $\mathrm{H}+\mathrm{Al}$ e foram calculadas a soma de bases, CTC e saturação por bases. As análises químicas de solo seguiram os métodos descritos por Raij et al. (2001). Por fim, foram calculadas as variações ( $\Delta=$ valor do atributo na amostragem final - valor na amostragem inicial) nos diversos atributos químicos do solo, ao longo de quatro safras, nas duas camadas de amostragem.

Em cada ciclo de produção, estimou-se a produtividade expressa em $\mathrm{kg} / \mathrm{planta}$ e $\mathrm{t} / \mathrm{ha}$, mediante a pesagem dos cachos na ocasião da colheita. Em amostras de 10 cachos por parcela experimental, foram avaliados a massa fresca, comprimento e largura dos cachos e bagas e número de bagas/cacho.

Os efeitos dos tratamentos sobre a fertilidade do solo, estimados por meio da diferença entre os valores dos atributos químicos observados entre a amostragem final e inicial $(\Delta)$, a produtividade e as características físicas dos cachos foram avaliados empregando-se o teste F. Para as situações nas quais se detectaram efeitos significativos dos tratamentos de adubação $(\mathrm{p}<0,10)$, foram ajustadas equações de regressão relacionando doses de $\mathrm{N}, \mathrm{P}_{2} \mathrm{O}_{5}$ ou $\mathrm{K}_{2} \mathrm{O}$ com 
as variáveis resposta.

\section{RESULTADOS E DISCUSSÃO}

Os teores de nutrientes disponíveis no solo, previamente à instalação do experimento, eram bastante elevados (Tabela 1). O teor de P disponível, segundo Raij et al. (1997), foi classificado como "muito alto" $\left(>60 \mathrm{mg} / \mathrm{dm}^{3}\right)$ nas duas profundidades amostradas, e o K trocável, como "alto" ( 3,1 a 6,0 $\mathrm{mmol} / \mathrm{dm}^{3}$ ), indicando uso excessivo de fertilizantes. Esta situação é comum em vinhedos da região de Louveira e Jundiaí, nos quais, de acordo com Tecchio et al. (2006), a adubação é feita frequentemente com base em orientações empíricas, utilizando-se de fertilizantes sem levar em conta a disponibilidade de nutrientes estimada pela análise de solo, e as necessidades das plantas.

A análise de variância dos efeitos dos tratamentos de adubação em alguns atributos químicos do solo, como o teor de matéria orgânica, $\mathrm{pH}$ $\left(\mathrm{CaCl}_{2}\right), \mathrm{P}, \mathrm{K}, \mathrm{Ca}, \mathrm{Mg}, \mathrm{H}+\mathrm{Al}$, soma de bases, CTC e saturação por bases, medidos em duas profundidades ( 0 a 20 e de 20 a $40 \mathrm{~cm}$ ) na amostragem final do experimento, evidenciou efeitos significativos $(\mathrm{p}<0,05)$ somente sobre a acidez, $\mathrm{P}$ e $\mathrm{K}$ disponíveis (Figuras 1 e 2). As demais variáveis $(\mathrm{Ca}, \mathrm{Mg}$ e $\mathrm{H}+\mathrm{Al})$ não foram afetadas pela adubação $(\mathrm{p}>0,05)$. $\mathrm{O}$ efeito da adubação com N, P e K em videira, na variação de alguns atributos químicos do solo ao longo de quatro safras (ago/05 a mar/08), expressa pelos $\Delta$ 's, seguiu a mesma tendência revelada pela análise de variância dos dados da amostragem final (Tabela 2).

$\mathrm{Na}$ falta de um método adequado para análise de $\mathrm{N}$ no solo, verificou-se o efeito da aplicação de $\mathrm{N}$, principalmente no aumento da acidez expresso pelo $\mathrm{pH}\left(\mathrm{CaCl}_{2}\right)$. A capacidade de acidificar o solo de alguns fertilizantes nitrogenados é conhecida e deriva de reações que produzem $\mathrm{H}^{+}$como a nitrificação e a perda de cátions para camadas mais profundas, acompanhando o ânion $\mathrm{NO}_{3}^{-}$(TISDALE et al., 1993). Ao final de quatro safras, o pH do solo diminuiu linearmente com o incremento nas doses de N, detectando-se este efeito até na camada de 20 a $40 \mathrm{~cm}$ (Figura 1A). Em média, cada $100 \mathrm{~kg} / \mathrm{ha}$ de $\mathrm{N}$ aplicados por safra causou redução de aproximadamente 0,65 unidade de $\mathrm{pH}$ na camada de 0 a $20 \mathrm{~cm}$ e de 0,66 unidade, de 20 a $40 \mathrm{~cm}$ (Tabelas 2 e 3). Este efeito sugere que áreas com aplicações contínuas de $\mathrm{N}$ na forma amídica ou amoniacal devam ser monitoradas, especialmente em relação à acidez do solo. Estes resultados são compatíveis com os descritos para outras frutíferas no Estado de São Paulo, como bananeira (SAES, 1995; TEIXEIRA et al., 2001), coqueiro (TEIXEIRA et al., 2005) e citros (SANCHES et al., 1999). Trabalhos na literatura relataram os problemas associados à redução do $\mathrm{pH}$ no solo, na cultura da videira. Marcelin (1974) verificou pouco desenvolvimento da videira e redução no crescimento radicular, em solos com $\mathrm{pH}$ menor que 5,0, relacionando-os à presença de $\mathrm{Al}^{3+}$ trocável. Himelrick (1991) avaliou o comportamento de nove cultivares de videira pelas suas tolerâncias em solos com pH 4,8 e 6,7 , observando que solos com $\mathrm{pH} 4,8$ ocasionaram redução na massa seca da parte aérea e das raízes, em todos os porta-enxertos, com exceção do 'Chardonnay' e 'White Riesling'. Bates et al. (2002) concluíram que as videiras 'Concord', cultivadas na região do Lago Erie (EUA), têm seu desenvolvimento máximo em solos com pH entre 5,0 e 6,0.

Aumentos nas doses de $\mathrm{P}$ tiveram efeito significativo nos teores de $\mathrm{P}$ disponível no solo (Figura 1B). A menor dose de $\mathrm{P}\left(60 \mathrm{~kg} / \mathrm{ha}\right.$ de $\left.\mathrm{P}_{2} \mathrm{O}_{5}\right)$ foi suficiente para promover seu acúmulo no solo ao longo das quatro safras, causando incrementos de 67 e $31 \mathrm{mg} / \mathrm{dm}^{3}$ nas camadas de 0 a 20 e de 20 a $40 \mathrm{~cm}$, respectivamente (Tabela 2). Nos tratamentos sem aplicação de $\mathrm{P}$, houve redução no teor de $\mathrm{P}$ disponível no solo em relação à situação inicial na camada de 0 a $20 \mathrm{~cm}\left(\Delta\right.$ Pdisponível $\left.=-21 \mathrm{mg} / \mathrm{dm}^{3}\right)$, mas mesmo assim o P disponível ainda foi classificado como muito alto (>60 mg/dm³ , segundo Raij et al., 1997). De 20 a $40 \mathrm{~cm}$, o teor de P manteve-se praticamente constante $\left(\Delta\right.$ Pdisponível $\left.=5 \mathrm{mg} / \mathrm{dm}^{3}\right)$, mesmo sem adubação fosfatada (Tabela 2). Estas observações indicam que a necessidade de $\mathrm{P}$ da videira seja relativamente pequena, especialmente diante dos elevados teores de $\mathrm{P}$ disponível no solo antes da instalação do experimento (Tabela 1). O uso de cama de aviário forneceu aproximadamente $170 \mathrm{~kg} / \mathrm{ha}$ de $\mathrm{P}$ em cada aplicação, o que provavelmente também contribuiu para que os teores de $\mathrm{P}$ disponível do solo se mantivessem aproximadamente constantes, mesmo sem a adubação fosfatada mineral. Teores altos de P no solo são frequentemente encontrados nos vinhedos da região de Louveira e Jundiaí. Tecchio (2005), num levantamento da fertilidade do solo em 40 vinhedos nessas regiões, constatou que a maior percentagem dos solos dos vinhedos encontrava-se com teores superiores a $60 \mathrm{mg} / \mathrm{dm}^{3}$, considerados muito altos, segundo Raij et al. (1997), sendo que teores de P acima de $300 \mathrm{mg} / \mathrm{dm}^{3}$ de solo foram encontrados em $50,6 \%$ das amostras. Tecchio (2005) atribuiu estes valores elevados à excessiva adubação fosfatada realizada anualmente pelos viticultores, a qual é muito superior às recomendações de adubação apresentadas por Terra et al. (1997). Em levantamentos nutricionais realizados por Gergoletti (1995), Regina et al. (1998) e Costa (1998) também foram verificados teores de 
P no solo bastante elevados em grande número de vinhedos amostrados, atribuindo estes resultados ao excesso de adubação fosfatada.

Os teores de K trocável do solo (Figura 2) aumentaram linearmente com o incremento nas doses de $\mathrm{K}$. Cada $100 \mathrm{~kg} / \mathrm{ha}$ de $\mathrm{K}_{2} \mathrm{O}$ aplicados por safra determinou incremento médio de $1,25 \mathrm{mmol} / \mathrm{dm}^{3}$ na camada de 0 a $20 \mathrm{~cm}$ (Tabela 2); de 20 a $40 \mathrm{~cm}$, cada $100 \mathrm{~kg} /$ ha de $\mathrm{K}_{2} \mathrm{O}$ implicou aumento médio de $1,6 \mathrm{mmol}_{\mathrm{c}} / \mathrm{dm}^{3}$, indicando possível lixiviação. Aumento na movimentação de K, no perfil do solo, proporcional à dose de $\mathrm{K}$ aplicada, também foi descrito por Ritchey (1982) num Latossolo VermelhoEscuro e por Teixeira et al. (2005) em área cultivada com coqueiro. A variação do teor de $\mathrm{K}$ disponível ao longo das quatro safras, nos tratamentos sem adubação potássica ( $\Delta$ Ktrocável próximo de zero), provavelmente se deveu aos elevados teores de $\mathrm{K}$ do solo existentes antes da instalação do experimento (Tabela 1). O aporte de aproximadamente $140 \mathrm{~kg} / \mathrm{ha}$ de K por aplicação de cama de aviário também deve ter contribuído com a manutenção do teor de $\mathrm{K}$ trocável do solo ao longo de quatro safras, mesmo sem a aplicação de $\mathrm{K}$ mineral. $\mathrm{O}$ excesso de potássio no solo pode ocasionar problemas na absorção de cálcio e magnésio em videiras (TAGLIAVANI et al., 1996).

Apesar dos efeitos significativos determinados pela adubação com N, P e K em alguns atributos químicos do solo, não foram observadas respostas na produção das videiras ao longo de quatro safras (Tabelas 4 e 5). A falta de resposta na produção à apli- cação de NPK em videira 'Niagara Rosada' também foi observada por Dal Bó (1992) em Santa Catarina. Provavelmente, não houve influência da adubação com N, P e K na produção e na qualidade dos cachos devido aos altos teores de nutrientes no solo, especificamente de $\mathrm{P}$ e de $\mathrm{K}$, encontrados no vinhedo previamente à implantação do experimento (Tabela 1). A elevada disponibilidade de nutrientes, que é uma condição comum nos cultivos de uva da região de Louveira e de Jundiaí,decorrente do intenso uso de fertilizantes ao longo dos anos, associada à utilização da cama de aviário, foi suficiente para suprir a demanda nutricional da videira nos quatro ciclos de produção. A falta de resposta da videira e os efeitos no solo da adubação com N, P e K, observados nos quatro ciclos de produção, permitem inferir que fração significativa dos nutrientes não tenha sido incorporada à biomassa das plantas, aumentando o risco de contaminação ambiental. Grant (2010) apresentou os problemas ambientais decorrentes do uso inadequado dos fertilizantes nitrogenados e fosfatados, destacando a eutrofização das águas e a contaminação dos mananciais por nitratos.

A aplicação de N, P e K nestes vinhedos com histórico de adubação intensa, sem levar em consideração a disponibilidade de nutrientes e as exigências da videira, implica prejuízo econômico ao produtor, eleva os teores de nutrientes no solo acima das necessidades da cultura, podendo, ainda, aumentar o risco de contaminação ambiental.

TABELA 1 - Atributos químicos de um solo cultivado com 'Niagara Rosada'. Valores médios para toda área em amostragem realizada na implantação do experimento (agosto/2005).

\begin{tabular}{ccccccccccc}
\hline Camada & $\mathrm{pH}(\mathrm{CaCl} 2)$ & $\mathrm{MO}$ & $\mathrm{P}_{\text {(resina) }}$ & $\mathrm{K}^{+}$ & $\mathrm{Ca}^{2+}$ & $\mathrm{Mg}^{2+}$ & $\mathrm{H}+\mathrm{Al}$ & $\mathrm{SB}$ & $\mathrm{T}$ & $\mathrm{V}$ \\
\hline$---\mathrm{cm}---$ & & $\mathrm{g} / \mathrm{dm}^{3}$ & $\mathrm{mg} / \mathrm{dm}^{3}$ & ---------------- & $\mathrm{mmol}_{\mathrm{c}} / \mathrm{dm}^{3}$ & ---------------- & $\%$ \\
$0-20$ & 5,3 & 18 & 81 & 3,4 & 33 & 9 & 20 & 45 & 64 & 69 \\
$20-40$ & 5,3 & 17 & 76 & 3,4 & 31 & 9 & 20 & 43 & 63 & 68 \\
\hline
\end{tabular}

${ }^{1} \mathrm{pH}$ do solo medido em solução de $\mathrm{CaCl}_{2} 0,01 \mathrm{~mol} / \mathrm{L}$, solo:solução = 1:2,5 (v/v); cátions trocáveis e P extraídos com resina trocadora de íons, segundo Raij et al. (2001). 
TABELA 2 - Alterações de $\mathrm{pH}(\Delta \mathrm{pH})$, dos teores de $\mathrm{P}(\Delta \mathrm{P})$ e de $\mathrm{K}(\Delta \mathrm{K})$ do solo em duas profundidades de amostragem, numa área cultivada com videira 'Niagara Rosada' submetida a doses de $\mathrm{N}$, $\mathrm{P}_{2} \mathrm{O}_{5}$ e $\mathrm{K}_{2} \mathrm{O}$. Louveira-SP.

\begin{tabular}{|c|c|c|c|c|c|c|c|}
\hline \multirow{2}{*}{ Nutriente } & \multirow[b]{2}{*}{ Dose } & \multicolumn{2}{|c|}{$\Delta^{(1)} \mathrm{pH}\left(\mathrm{CaCl}_{2}\right)$} & \multicolumn{2}{|c|}{$\Delta \mathrm{P}(\mathrm{resina})$} & \multicolumn{2}{|c|}{$\Delta \mathrm{K}$} \\
\hline & & 0 a $20 \mathrm{~cm}$ & 20 a $40 \mathrm{~cm}$ & 0 a $20 \mathrm{~cm}$ & 20 a $40 \mathrm{~cm}$ & 0 a $20 \mathrm{~cm}$ & 20 a $40 \mathrm{~cm}$ \\
\hline & & & & \multicolumn{2}{|c|}{$\mathrm{mg} / \mathrm{dm}^{3}$} & \multicolumn{2}{|c|}{$\mathrm{mmolc} / \mathrm{dm}^{3}$} \\
\hline \multirow{4}{*}{$\mathrm{N}$} & 0 & $-0,08$ & $-0,11$ & 92 & 67 & 1,8 & 1,1 \\
\hline & 65 & $-0,61$ & $-0,46$ & 104 & 27 & 1,3 & 0,8 \\
\hline & 130 & $-0,85$ & $-0,74$ & 75 & 33 & 1,0 & 0,1 \\
\hline & 195 & $-1,40$ & $-1,45$ & 116 & 46 & 0,4 & 0,1 \\
\hline Efeito $^{(2)}$ & & $\mathrm{L}^{* *}$ & $\mathrm{~L}^{* *}$ & NS & NS & NS & NS \\
\hline \multirow{4}{*}{$\mathrm{P}$} & 0 & $-0,69$ & $-0,58$ & -21 & 5 & 1,4 & 0,7 \\
\hline & 60 & $-0,76$ & $-0,80$ & 67 & 31 & 1,2 & 0,6 \\
\hline & 120 & $-0,60$ & $-0,61$ & 147 & 63 & 0,9 & 0,6 \\
\hline & 180 & $-0,89$ & $-0,78$ & 194 & 74 & 0,9 & 0,1 \\
\hline Efeito & & NS & NS & $\mathrm{L}^{* *}$ & $\mathrm{~L}^{* *}$ & NS & NS \\
\hline \multirow{4}{*}{ K } & 0 & $-0,54$ & $-0,65$ & 79 & 47 & 0,0 & $-0,5$ \\
\hline & 45 & $-0,65$ & $-0,54$ & 114 & 42 & 1,3 & 0,1 \\
\hline & 90 & $-0,78$ & $-0,75$ & 117 & 44 & 1,3 & 0,7 \\
\hline & 135 & $-0,98$ & $-0,83$ & 78 & 40 & 1,9 & 1,8 \\
\hline Efeito & & NS & NS & NS & NS & $\mathrm{L}^{*}$ & $\mathrm{~L}^{* *}$ \\
\hline
\end{tabular}

(1) $\Delta=$ valor obtido na amostragem final valor inicial; ${ }^{(2)}$ Efeito: $\mathrm{L}=$ efeito linear, $\mathrm{Q}=$ efeito quadrático, $\mathrm{NS}=\mathrm{p}>0,05, *=0,01<\mathrm{p}<0,05$ $\mathrm{e}^{* *}=\mathrm{p}<0,01$

TABELA 3 - Efeito da aplicação de N, P e K (doses em kg/ha de $\mathrm{N}, \mathrm{P}_{2} \mathrm{O}_{5}$ e $\mathrm{K}_{2} \mathrm{O}$ ) nas alterações de $\mathrm{pH}(\Delta \mathrm{pH})$ e dos teores de $\mathrm{P}(\Delta \mathrm{P})$ e de $\mathrm{K}(\Delta \mathrm{K})$, após quatro safras de 'Niagara Rosada', avaliado em duas profundidades de amostragem de solo. Louveira-SP.

\begin{tabular}{|c|c|c|c|c|c|}
\hline \multicolumn{2}{|l|}{$\Delta^{(1)} \mathrm{pH} \mathrm{CaCl}{ }_{2}$} & \multicolumn{2}{|c|}{$\Delta$ Presina $\left(\mathrm{mg} / \mathrm{dm}^{3}\right)$} & \multicolumn{2}{|c|}{$\Delta \mathrm{K}\left(\mathrm{mmol}_{\mathrm{c}} / \mathrm{dm}^{3}\right)$} \\
\hline Função & $\mathrm{R}^{2}$ & Função & $\mathrm{R}^{2}$ & Função & $\mathrm{R}^{2}$ \\
\hline ---------.. & ---. & 0 a 20 & ----- & --------------' & ------ \\
\hline$\Delta \mathrm{pH}=-0,102-6,48 \times 10^{-3} \mathrm{~N}$ & 0,97 & $\Delta \mathrm{P}=-12,30+1,21 \mathrm{P}$ & 0,97 & $\Delta \mathrm{K}=0,256+0,013 \mathrm{~K}$ & 0,75 \\
\hline$\Delta \mathrm{pH}=-0,047-6,60 \times 10^{-3} \mathrm{~N}$ & 0,93 & $\Delta \mathrm{P}=7,46+0,40 \mathrm{P}$ & 0,95 & $\Delta \mathrm{K}=-0,580+0,016 \mathrm{~K}$ & 0,95 \\
\hline
\end{tabular}

${ }^{(1)} \Delta=$ valor obtido na amostragem final valor inicial.

TABELA 4 - Resultados médios da produtividade, número de cachos por planta e de bagas/cacho de videira 'Niagara Rosada' submetida a doses de $\mathrm{N}, \mathrm{P}_{2} \mathrm{O}_{5}$ e $\mathrm{K}_{2} \mathrm{O}$, em quatro ciclos de produção. Louveira, 2005 a 2007.

\begin{tabular}{cccccc}
\hline Nutriente & Dose & \multicolumn{2}{c}{ Produtividade } & Cachos por planta & Bagas por cacho \\
\hline & & $\mathrm{kg} / \mathrm{planta}$ & $\mathrm{t} / \mathrm{ha}$ & & \\
$\mathrm{N}$ & 0 & 2,1 & 13,9 & 10,7 & 45,8 \\
& 65 & 1,8 & 12,1 & 9,2 & 45,7 \\
& 130 & 2,0 & 13,3 & 10,2 & 46,4 \\
\hline Efeito & 195 & 2,0 & 13,2 & 9,9 & 51,7 \\
\hline $\mathrm{P}$ & 0 & $\mathrm{NS}$ & $\mathrm{NS}$ & $\mathrm{NS}$ & $\mathrm{NS}$ \\
$\mathrm{P}$ & 60 & 1,9 & 12,5 & 9,5 & 49,2 \\
& 120 & 2,1 & 13,7 & 10,4 & 47,7 \\
& 180 & 2,0 & 12,9 & 9,9 & 46,8 \\
\hline Efeito & 2,1 & 13,5 & 10,1 & 45,8 \\
\hline & & $\mathrm{NS}$ & $\mathrm{NS}$ & $\mathrm{NS}$ & $\mathrm{NS}$ \\
$\mathrm{K}$ & 0 & 1,9 & 12,6 & 9,5 & 44,1 \\
& 45 & 2,2 & 14,6 & 10,6 & 49,7 \\
& 90 & 1,9 & 12,7 & 9,9 & 49,7 \\
\hline Efeito & 135 & 1,9 & 12,7 & 9,9 & 46,0 \\
\hline
\end{tabular}

NS: efeito não significativo a $5 \%$ de probabilidade $(\mathrm{p}>0,05)$ 
TABELA 5 - Resultados médios de massa, comprimento e largura de cachos e de bagas de videira 'Niagara Rosada' submetida a doses de $\mathrm{N}, \mathrm{P}_{2} \mathrm{O}_{5}$ e $\mathrm{K}_{2} \mathrm{O}$, em quatro ciclos de produção. Louveira-SP.

\begin{tabular}{|c|c|c|c|c|c|c|c|}
\hline \multirow[b]{2}{*}{ Nutriente } & \multirow[b]{2}{*}{ Dose } & \multicolumn{3}{|c|}{ Cacho } & \multirow[b]{2}{*}{$\begin{array}{l}\text { Massa } \\
\text { fresca }\end{array}$} & \multicolumn{2}{|c|}{ Bagas } \\
\hline & & Massa fresca & Comp. & Larg. & & Comp. & Larg. \\
\hline \multirow{5}{*}{$\mathrm{N}$} & & $\mathrm{g}$ & $\mathrm{cm}$ & $\mathrm{cm}$ & $\mathrm{g}$ & $\mathrm{cm}$ & $\mathrm{cm}$ \\
\hline & 0 & 195,4 & 12,6 & 7,4 & 4,9 & 8,8 & 7,7 \\
\hline & 65 & 195,1 & 12,4 & 7,4 & 4,8 & 8,9 & 7,7 \\
\hline & 130 & 194,1 & 12,8 & 7,4 & 4,9 & 8,9 & 7,7 \\
\hline & 195 & 206,5 & 13,1 & 7,6 & 4,8 & 8,6 & 7,7 \\
\hline \multirow[t]{2}{*}{ Efeito } & & NS & NS & NS & NS & NS & NS \\
\hline & 0 & 195,4 & 12,8 & 7,5 & 4,7 & 8,9 & 7,7 \\
\hline \multirow[t]{3}{*}{$\mathrm{P}$} & 60 & 197,3 & 12,9 & 7,4 & 4,9 & 8,9 & 7,7 \\
\hline & 120 & 197,2 & 12,9 & 7,4 & 4,8 & 8,8 & 7,7 \\
\hline & 180 & 201,3 & 12,4 & 7,5 & 4,9 & 8,6 & 7,7 \\
\hline \multirow[t]{2}{*}{ Efeito } & & NS & NS & NS & NS & NS & NS \\
\hline & 0 & 195,9 & 12,4 & 7,2 & 4,7 & 8,4 & 7,6 \\
\hline \multirow[t]{3}{*}{$\mathrm{K}$} & 45 & 206,2 & 12,8 & 7,6 & 4,9 & 9,1 & 7,8 \\
\hline & 90 & 194,8 & 12,9 & 7,5 & 4,9 & 8,8 & 7,7 \\
\hline & 135 & 194,5 & 12,8 & 7,5 & 4,8 & 8,8 & 7,8 \\
\hline Efeito & & NS & NS & NS & NS & NS & NS \\
\hline
\end{tabular}

NS: efeito não significativo a $5 \%$ de probabilidade $(\mathrm{p}>0,05)$

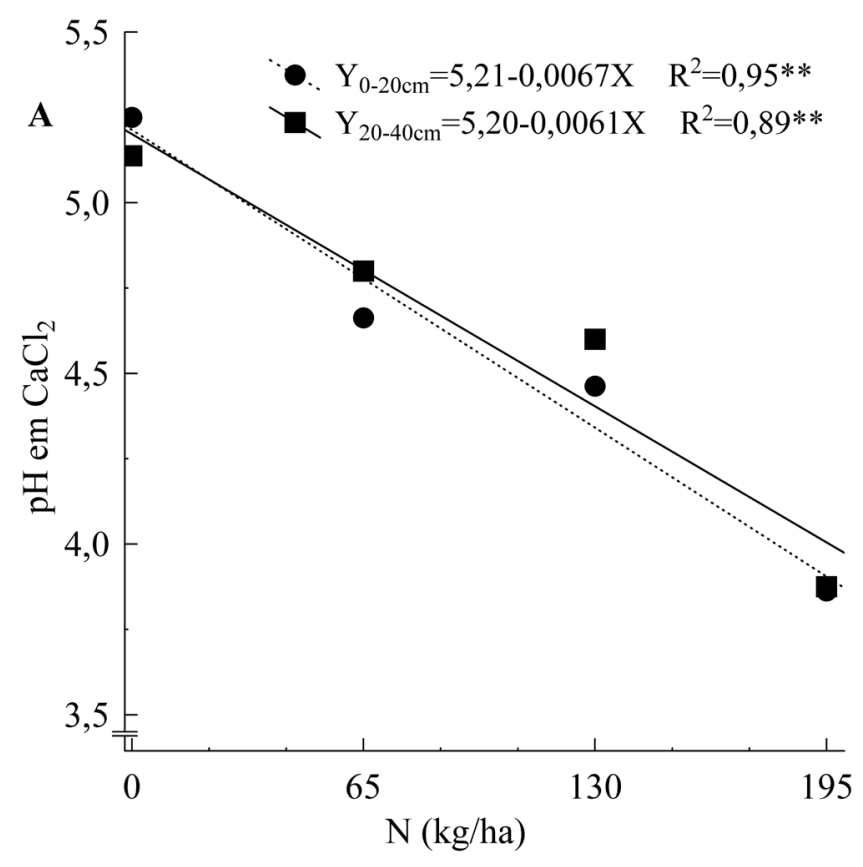

continua 


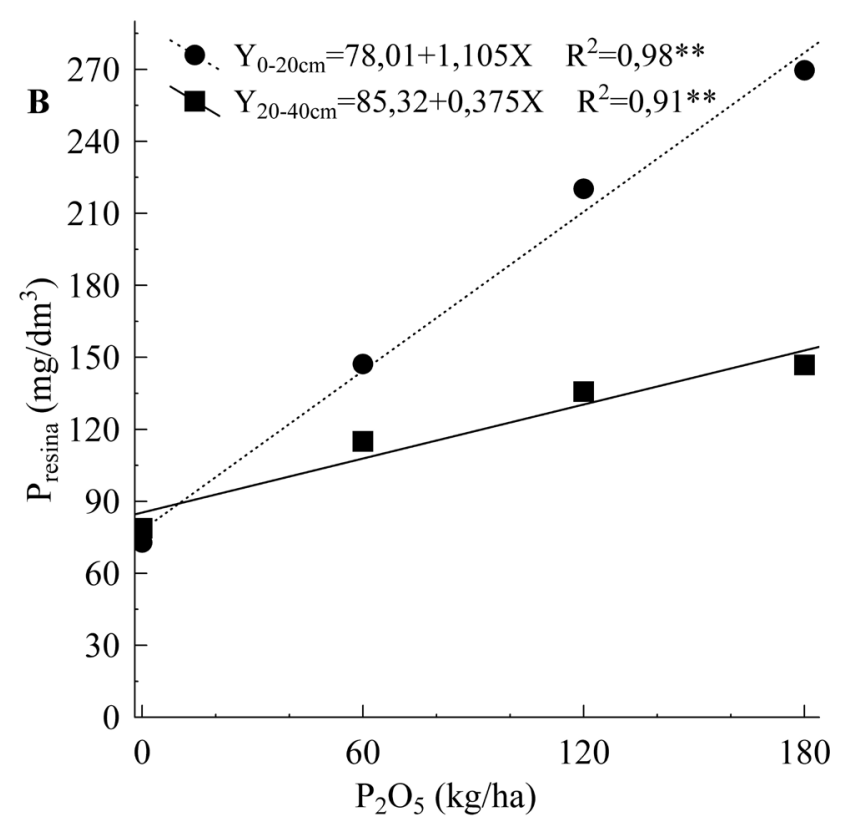

FIGURA 1 - Efeito da adubação nitrogenada em videira no pH do solo (A) e da adubação fosfatada no teor de P disponível (B), após quatro safras. .**significativo $(\mathrm{p}<0,01)$.

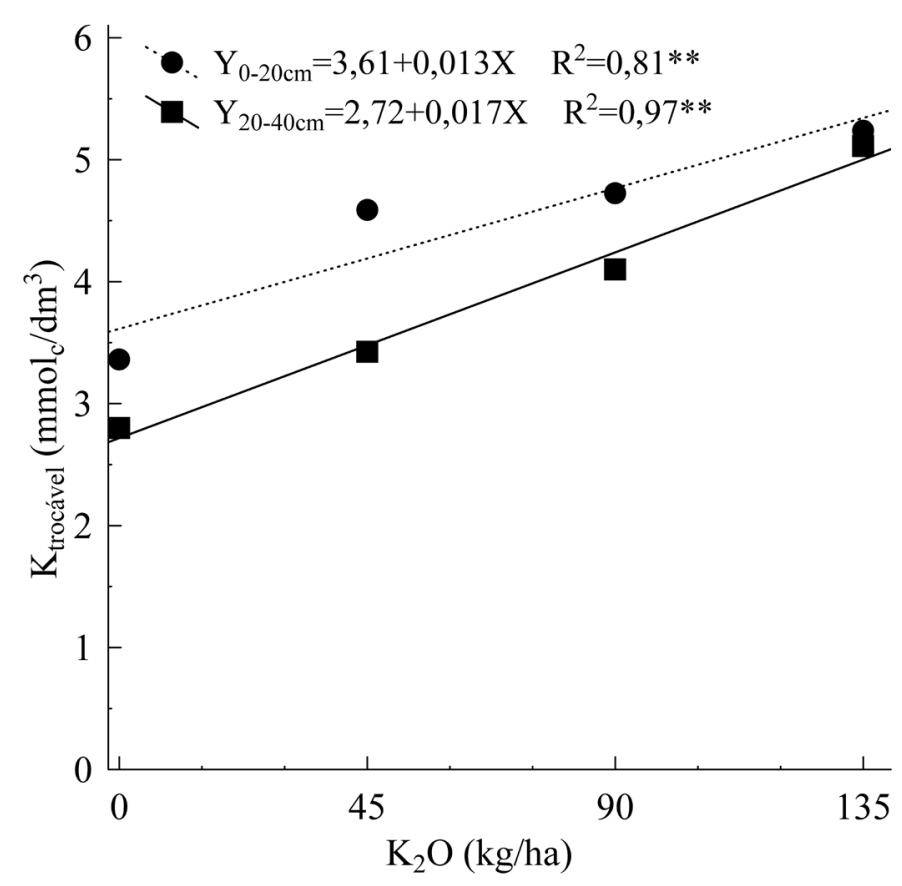

FIGURA 2 - Efeito da adubação potássica em videira no teor de K trocável do solo, após quatro safras. ${ }^{*}$ significativo $(\mathrm{p}<0,01)$ 


\section{CONCLUSÕES}

1-A adubação nitrogenada em videira determina incrementos significativos na acidez do solo, após quatro ciclos de produção.

2-A adubação com N, P e K aumenta a disponibilidade destes nutrientes no solo, entretanto não há resposta na produtividade e na qualidade dos cachos da videira 'Niagara Rosada'. Na condição de alta disponibilidade de $\mathrm{P}$ e $\mathrm{K}$ no solo, comum nos vinhedos da região de Jundiaí, a aplicação destes nutrientes implica gasto desnecessário, podendo, ainda, potencializar a contaminação ambiental

\section{REFERÊNCIAS}

BATES, T.R.; DUNST, R.M.; TAFT, T.; VERCANT, $M$. The vegetative response of 'Concord' grapevines to soil pH. Hortscience, Stanford, v.37, n. 6, p. 890893, 2002.

BRAGA, F. G. Cultura da uva Niágara Rosada. São Paulo: Nobel, 1988. 66p.

CONRADIE, W. J.; SAAYMAN, D. Effects of LongTerm Nitrogen, Phosphorus, and Potassium Fertilization on Chenin blanc Vines. I. Nutrient Demand and Vine Performance. American Journal of Enology and Viticulture, Davis, v. 40, n. 2, p. 85-90, 1989a.

CONRADIE, W. J.; SAAYMAN, D. Effects of LongTerm Nitrogen, Phosphorus, and Potassium Fertilization on Chenin blanc Vines. II. Leaf Analyses and Grape Composition. American Journal of Enology and Viticulture, Davis, v. 40, n. 2, p. 91-98, 1989 b.

COSTA, F. Avaliação do estado nutricional da videira cultivar Itália em três estádios de desenvolvimento, na região de JundiaíSP, utilizando o método DRIS. 1998. 91 f. Dissertação (Mestrado em Agronomia/Fitotecnia) Escola Superior de Agricultura "Luiz de Queiroz", Universidade de São Paulo, Piracicaba, 1998.

DAL BÓ, M.A. Efeito da adubação NPK na produção, qualidade da uva e nos teores foliares de nutrientes da videira. Revista Brasileira de Fruticultura, Jaboticabal, v. 14, n. 2, p. 189- 194, 1992.

FRÁGUAS, J. C.; REGINA, M. A.; ALVARENGA, A. A.; ABRAHÃO, E.; ANTUNES, L. E. C.; FADINI, M. A. M. Calagem e adubação para videira e fruteiras de clima temperado. Belo Horizonte: Epamig, 2002. 44 p. (Boletim Técnico, 65).
FREGONI, M. Nutrizione e fertilizzazione della vite. Bologna: Edagricole, 1980. 418 p.

Gergoletti, I. F. Avaliação do estado nutricional da videira cultivar Itália em três estádios de desenvolvimento, em São Miguel Arcanjo-SP, utilizando o método DRIS. 1995. 84 f. Tese (Doutorado em Agronomia/Solos e Nutrição de Plantas) - Escola Superior de Agricultura "Luiz de Queiroz”, Universidade de São Paulo, Piracicaba, 1995.

GRANT, C. Effects of nitrogen and phosphorus fertilizers on the environment. In: PROCHNOW, L.I.; CASARIN, V.; STIPP, S.R. (Ed.). Boas práticas para uso eficiente de fertilizantes: contexto mundial e técnicas de suporte. Piracicaba: IPNI, 2010. p.4390 .

HIMELRICK, D.G. Growth and nutritional responses of nine grape cultivars to low soil $\mathrm{pH}$. HortScience, Stanford, v.26, n.3, p.269-271, 1991.

HIBERT, G.; SOYER, J. P.; MOLOT, C.; GIRAUDON, J.; MILIN, S.; GAUDILLERE, J. P. Effects of nitrogen supply on must quality and anthocyanin accumulation in berries of cv. Merlot. Vitis, Siebeldingen, v. 42, n. 2,p. 69-76, 2003.

INSTITUTO DE ECONOMIA AGRÍCOLA. Produção. Disponível em: $<$ http:// www.iea.sp.gov. br.>. Acesso em: 16 jun.2010.

LANYON, D.M.; CASS, A.; HANSEN, D. The effect of soil properties on vine performance. Australia: CSIRO, 2004. 54p. (Land Water Technical Report, 34/04)

LINSENMEIER, A.W.; LOOS, U.; LÖHNERTZ, O. Must Composition and Nitrogen Uptake in a Long-term Trial as Affected by Timing of Nitrogen Fertilization in a Cool-Climate Riesling Vineyard. American Journal of Enology and Viticulture, Davis, v. 59, n. 3, p. 255-264, 2008.

MARCELIN, H. La vigne dans les sols acides de Roussillon. Progres Agricole et Viticole, Montpellier, v.91, n.18, p.570-576, 1974.

NEILSEN, G.H.; NEILSEN, D.; BOWEN, P.; BOGDANOFF, C.; USHER, K. Effect of Timing, Rate, and Form of N Fertilization on Nutrition, Vigor, Yield, and Berry Yeast-Assimilable N of Grape. American Journal of Enology and Viticulture, Davis, v. 61, n. 3, p. 327-336, 2010. 
OLIVEIRA, J.B.; CAMARGO, M.N.; ROSSI, M. ; CALDERANO FILHO, B. Mapa pedológico do Estado de São Paulo: legenda expandida. Campinas: Instituto Agronômico, 1999. 64p.

RAIJ, B. van; ALCARDE, J.C.; CANTARELLA, H.; QuAGGIO, J.A. (Ed.). Análise química para avaliação da fertilidade de solos tropicais. Campinas: IAC, 2001. 285p.

RAIJ, B. van; CANTARELLA, H.; QUAGGIO, J.A.; FURLANI, A.M.C. Recomendações de adubação e calagem para o Estado de São Paulo. 2. ed. Campinas: IAC, 1997. (Boletim Técnico, 100)

REGINA, M.A.; ALVARENGA, A. A.; CHALFUN, N. J. \& CHALFUN JÚNIOR, A. Levantamento nutricional e diagnóstico agronômico dos vinhedos de caldas. Revista Brasileira de Fruticultura, Jaboticabal, v. 20, n. 1, p. 15-20, 1998.

REUVENI, M.; NAOR, A.; REUVENI, R.; SHIMONI, M.; BRAVDO, B. The Influence of NPK Fertilization Rates on Susceptibility to Powdery Mildew of Field-Grown Winegrapes. Journal of Small Fruit \& Viticulture, Beltsville, v.2, p. 31-41, 1994.

RITCHEY, K.D. O potássio nos oxissolos e ultissolos dos trópicos úmidos. Piracicaba: Instituto da Potassa e Fosfato/Instituto Internacional da Potassa, 1982. 69p. (Boletim Técnico, 7)

SAES, L.A. Resposta da bananeira "Nanicão" à calagem na região do Vale do Ribeira, 1995. 82f. Dissertação (Mestrado em Agronomia) Escola Superior de Agricultura Luiz de Queiroz, Universidade de São Paulo, Piracicaba, 1995.

SANCHES, A.C.; SILVA, A.P.; TORMENA, C.A.; RIGOLIN, A.T. Impacto do cultivo de citros em propriedades químicas, densidade do solo e atividade microbiana de um podzólico vermelho-amarelo. Revista Brasileira de Ciência do Solo, Viçosa, $\mathrm{MG}$, v.23, p.91-99, 1999.

SHARMA, J.; SHIKHAMANY, S. D.; SINGH, R. K. Studies on inward leaf curl disorder in grape. Indian Journal of Horticulture, Lucknow, v. 60, n. 3, p. 236-238, 2003.

TAGLIAVANI, M. ; STEFFENS, D. ; PELLICONI, F. La carenza di potassio nei vigneti della Romagna. Vignevini, Bologna, v. 23, n. 4, p. 41-46, 1996.

TECCHIO, M.A. Levantamento nutricional e diagnóstico agronômico da videira 'Niagara Rosada' em Jundiaí e Louveira-SP. 2005. 109 f. Tese (Doutorado em Horticultura) - Faculdade de Ciências Agronômicas, Universidade Estadual Paulista, Botucatu, 2005.

TECCHIO, M.A.; PIRES, E.J.P.; TERRA, M.M.; GRASSI FILHO, H.; CORRÊA, J.C.; IDE VIEIRA, C.R.Y. Correlação entre a produtividade e os resultados de analise foliar e de sol oem vinhedos de Niagara Rosada. Ciência e Agrotecnologia, Lavras, v. 30, n. 6, p. 1056-1064, 2006.

TEIXEIRA, L.A.J.; BATAGLIA, O.; BUZETTI, S.; FURLANI JR., E. Adubação com NPK em coqueiroanão-verde (Cocos nucifera L.) - atributos químicos do solo e nutrição da planta. Revista Brasileira de Fruticultura, Jaboticabal, v.27, n.1, p.115-119, 2005.

TEIXEIRA, L.A.J.; NATALE, W.; RUGGIERO, C. Alterações em alguns atributos químicos do solo decorrentes da irrigação e adubação nitrogenada e potássica em bananeira após dois ciclos de cultivo. Revista Brasileira de Fruticultura, Jaboticabal, v.23, n.3, p.684-689, 2001.

TERRA, M.M. Nutrição, calagem e adubação. In: POMMER, C.V. Uva: tecnologia de produção, póscolheita, mercado. Porto Alegre: Cinco Continentes, 2003. cap. 7, p. 405-476.

TERRA, M.M. Seis anos de experimentação de adubação (NPK) em videira cultivar Niagara Rosada vegetando em um solo podzolizado, Indaiatuba, SP. 1989. 158 f. Tese (Doutorado em Agronomia) - Escola Superior de Agricultura "Luiz de Queiroz", Universidade de São Paulo, Piracicaba, 1990.

TERRA, M.M.; TECCHIO, M.A.; PIRES, E.J.; TEIXEIRA, L.A.J. Uvas comuns para mesa e vinho. In: RAIJ, B. van; CANTARELLA, H.; QUAGGIO, J.A.; FURLANI, A.M.C. Recomendações de adubação e calagem para o Estado de São Paulo. 2.ed. Campinas: Instituto Agronômico/Fundação IAC, 1997. p.152-153. (Boletim Técnico, 100)

TISDALE, S.L.; NELSON, W.; BEATON, J.D.; HAVLIN, J.L. Soil fertility and fertilizers. 5.ed. New York: Macmillan Publishing Company, 1993. $648 p$.

TONIETTO, J. Diagnóstico nutricional das videiras Isabel e Concord através da análise foliar. Revista Brasileira de Fruticultura, Jaboticabal, v. 16, n. 1, p. 185-194, 1994. 